\title{
Short-Term Outcomes of Lower Trapezius Tendon Transfer With Achilles Allograft for Irreparable Posterosuperior Rotator Cuff Tears
}

\author{
Michael A. Stone, M.D., Liam T. Kane, B.S., Jason C. Ho, M.D., and Surena Namdari, M.D.
}

\begin{abstract}
Purpose: To evaluate functional outcomes, return to work, and reoperations associated with lower trapezius tendon transfer (LTT) for irreparable rotator cuff tears. Methods: This is a retrospective study performed by a single surgeon with minimum l-year follow-up. LTT was performed using an open ( $\mathrm{n}=9 ; 60 \%)$ or arthroscopically assisted $(\mathrm{n}=6$, $40 \%$ ) technique. Outcomes included failure rate, range of motion, satisfaction, return to work, and pre- and postoperative functional scores, as well as American Shoulder and Elbow Surgeons score, Single Assessment Numeric Evaluation, and the Simple Shoulder Test. Results: Fifteen patients were included. LTT was performed using an open or arthroscopically assisted technique. Mean age was 52 (range 31-62 years), 13 (92.9\%) were manual laborers, and 9 (60\%) had a worker's compensation claim. Three patients $(20 \%)$ underwent conversion to reverse shoulder arthroplasty. Of the remaining 12 patients, there were significant improvements in American Shoulder and Elbow Surgeons, Single Assessment Numeric Evaluation, and Simple Shoulder Test at 24.1 \pm 9.6 (range 12-38.5) months. Active forward elevation, abduction, and external rotation were all significantly improved. Postoperative satisfaction ratings indicated $67 \%$ of the revision-free cohort was "very satisfied" and 33\% was "somewhat satisfied" with their outcome. Seven (50\%) returned to full duty, $4(28.6 \%)$ returned to modified duty, and $3(21.4 \%)$ were unable to return to work. Two patients (open techniques) underwent a superficial wound debridement for seroma and wound breakdown. Conclusions: LTT results in successful clinical outcomes with a high rate of return to work in a challenging patient population. However, only $67 \%$ of patients rated themselves as "very satisfied," and $20 \%$ of patients were revised to reverse shoulder arthroplasty before 1 year. Limited preoperative active forward elevation $\left(<90^{\circ}\right)$ appears to predict poor functional results and risk for reoperation. Level of Evidence: Level IV, therapeutic case series.
\end{abstract}

$\mathbf{I}$ rreparable rotator cuff tears (IRCTs) can cause significant deficits in function and are challenging to treat. There are a number of treatment options for these

From the Rothman Orthopaedic Institute at Thomas Jefferson University Hospital, Philadelphia, Pennsylvania, U.S.A.

The authors report the following potential conflicts of interest or sources of funding: S.N. reports other from Arthrex, DJO Surgical, Flexion Therapeutics, Force Therapeutics, Integra LifeXciences, Miami Device Solutions, Parvizi Surgical Solutions, Synthes, Zimmer Biomet, Saunders Mosby Elsevier, RubiconMD, MD Live, and MD Valuate, outside the submitted work. Full ICMJE author disclosure forms are available for this article online, as supplementary material.

Investigation performed at the Rothman Orthopaedic Institute, Thomas Jefferson University Hospital, Philadelphia, PA 19107.

Received March 6, 2020; accepted August 18, 2020.

Address correspondence to Surena Namdari, M.D., M.Sc., Associate Professor of Orthopaedic Surgery, Rothman Orthopaedic Institute, Thomas Jefferson University, Shoulder $\theta$ Elbow Surgery, 925 Chestnut St., 5th floor, Philadelphia, PA 19107.E-mail: Surena.namdari@rothmanortho.com

(C) 2020 THE AUTHORS. Published by Elsevier Inc. on behalf of the Arthroscopy Association of North America. This is an open access article under the CC BY-NC-ND license (http://creativecommons.org/licenses/by-nc-nd/4.0/).

2666-061X/20238

https://doi.org/10.1016/j.asmr.2020.08.004 tears, which include rotator cuff debridement, partial rotator cuff repair, biceps tenotomy or tenodesis, tendon transfer, superior capsule reconstruction, and reverse shoulder arthroplasty (RSA). ${ }^{1-6}$ In patients with goals of improved strength and/or return to manual labor, tendon transfer is commonly considered. The latissimus dorsi tendon transfer was initially described by Gerber ${ }^{7}$ with successful clinical results; however, Iannotti et al. $^{8}$ demonstrated that the latissimus uncommonly fired in-phase during external rotation after transfer. Because the natural force vector of the lower trapezius muscle better recreates the pull of the insufficient or absent infraspinatus as compared with the latissimus dorsi tendon, ${ }^{9,10}$ lower trapezius tendon transfer (LTT) has been proposed as an alternative option for irreparable posterosuperior rotator cuff tears. $^{11,12}$

There is currently a single study by Elhassan et al. ${ }^{11}$ describing clinical results of LTT for IRCTs using an acromial osteotomy for exposure. Several articles have since been published describing a mini-open technique and an arthroscopic-assisted technique. ${ }^{12-14}$ The 
purpose of this study is to evaluate functional outcomes, return to work, and reoperations associated with LTT for IRCTs. The primary outcome of interest for this study was to determine survivorship of the LTT for treatment of IRCT. Secondary outcomes included functional outcome scores, improvements in pain, range of motion (ROM), and ability to return to work. We hypothesized improved clinical outcomes and low rates of conversion to RSA in patients undergoing LTT for IRCT.

\section{Methods}

\section{Study Design}

Institutional review board approval (protocol \# 18D.437 by Thomas Jefferson University institutional review board) was obtained. This was a retrospective review of all LTT for IRCT performed by the senior author (S.N.) at a single institution from April 2014 to June 2018. Inclusion criteria included a massive, IRCT as determined by preoperative magnetic resonance imaging and confirmed intraoperatively, age $>18$ years, and normal function of the lower trapezius tendon. Exclusion criteria included any patients with active infection, nerve injury, severe glenohumeral arthritis, cuff tear arthropathy (Hamada grades IV and V), and concurrent or previous arthroplasty of the ipsilateral shoulder. Fifteen patients were included for analysis and all underwent either an open or arthroscopicassisted LTT.

\section{Data Variables}

Patient demographics including age, sex, body mass index, smoking status, previous surgeries, occupation, and worker's compensation claims were recorded (Table 1). Occupations were classified as laborintensive or nonlabor-intensive on the basis of the

Table 1. Patient Demographics

\begin{tabular}{lc}
\hline \multicolumn{1}{c}{ Variable } & $\begin{array}{c}\text { Mean } \pm \text { SD (range) or } \\
\mathrm{n}(\%)(\mathrm{n}=15)\end{array}$ \\
\hline Age & $52 \pm 7.3($ range $31-62)$ \\
Sex & $14(93.3 \%)$ \\
Male & $1(7.7 \%)$ \\
Female & $24.1 \pm 9.6($ range $12-38.5)$ \\
Follow-up, mo & $3(20.0 \%)$ \\
Smoking status & $12(80.0 \%)$ \\
$\quad$ Smoker & $1.3($ range $0-5)$ \\
Nonsmoker & $14(93.3 \%)$ \\
Previous surgeries, no. & $9(60 \%)$ \\
Previous rotator cuff surgery & $13(86.7 \%)$ \\
Worker's compensation claim & $9(60.0 \%)$ \\
Manual laborer & $6(40.0 \%)$ \\
Surgery type & \\
$\quad$ Open &
\end{tabular}

patients' self-classifications. Preoperative (within 6 months before surgery) and postoperative (minimum 12 months) active forward elevation (FE), abduction, and external rotation (ER) measurements were collected from the medical record. All ROM data were collected and recorded by the treating surgeon (S.N.), and a goniometer was not routinely used. Preoperative visual analog scale pain score, American Shoulder and Elbow Surgeons score, Simple Shoulder Test, Single Assessment Numeric Evaluation scores, and Veterans Rand Health Survey were collected. Patients were contacted at a minimum of 1 year postoperatively to obtain the same scores. Return to work, rates of conversion to RSA, and complications also were determined.

\section{Surgical Technique}

The cohort consisted of a combination of either open or arthroscopic-assisted LTT. Patients were placed in the lateral decubitus position for the open procedures and the beach chair position for the arthroscopicassisted transfer. For the open procedure, the indirect (medial) approach was performed. The incision was made $2 \mathrm{~cm}$ medial and parallel to the medial border of the scapula, extending $5 \mathrm{~cm}$ from the scapular spine (Fig 1A). The fascia overlying the muscle of the lower trapezius was incised, and the muscle was identified and dissected toward its insertion on the medial spine of the scapula. Finally, the tendon was peeled off its insertion as lateral as possible to maximize tendon length. To mobilize the muscle, dissection was bluntly performed between the lower and middle trapezius. An Achilles allograft was then sized and sutured to the native lower trapezius via a Pulver-Taft weave with multiple nonabsorbable sutures (Fig 1B). A second incision was then made approximating the junction between the anterior and middle deltoid. This interval was developed, and the greater tuberosity was prepared with a bur. The graft was then shuttled from the posterior wound and stitched with multiple locking stitches from two 5.5-mm (Arthrex, Naples, FL) suture anchors placed medially on the footprint. A double-row construct was creating using two 4.75 knotless (Arthrex) suture anchors placed laterally.

For the arthroscopic-assisted transfer, a direct approach to the lower trapezius was performed. A $4-\mathrm{cm}$ skin incision was made parallel to the scapular spine, centered on its medial side (Fig 2A). After identifying the LT tendon, its attachment on the spine of the scapula was released as distal as possible and bluntly mobilized. A diagnostic arthroscopy was then performed. After treatment of any concomitant pathology, the tuberosity was prepared using an arthroscopic burr and two 5.5-mm suture anchors were placed at the medial aspect of the greater tuberosity. Under the 
Fig 1. (A) Medial incision for an indirect approach of a patient in the lateral decubitus position (right shoulder). (B) Achilles tendon graft after repair to the lower trapezius tendon.
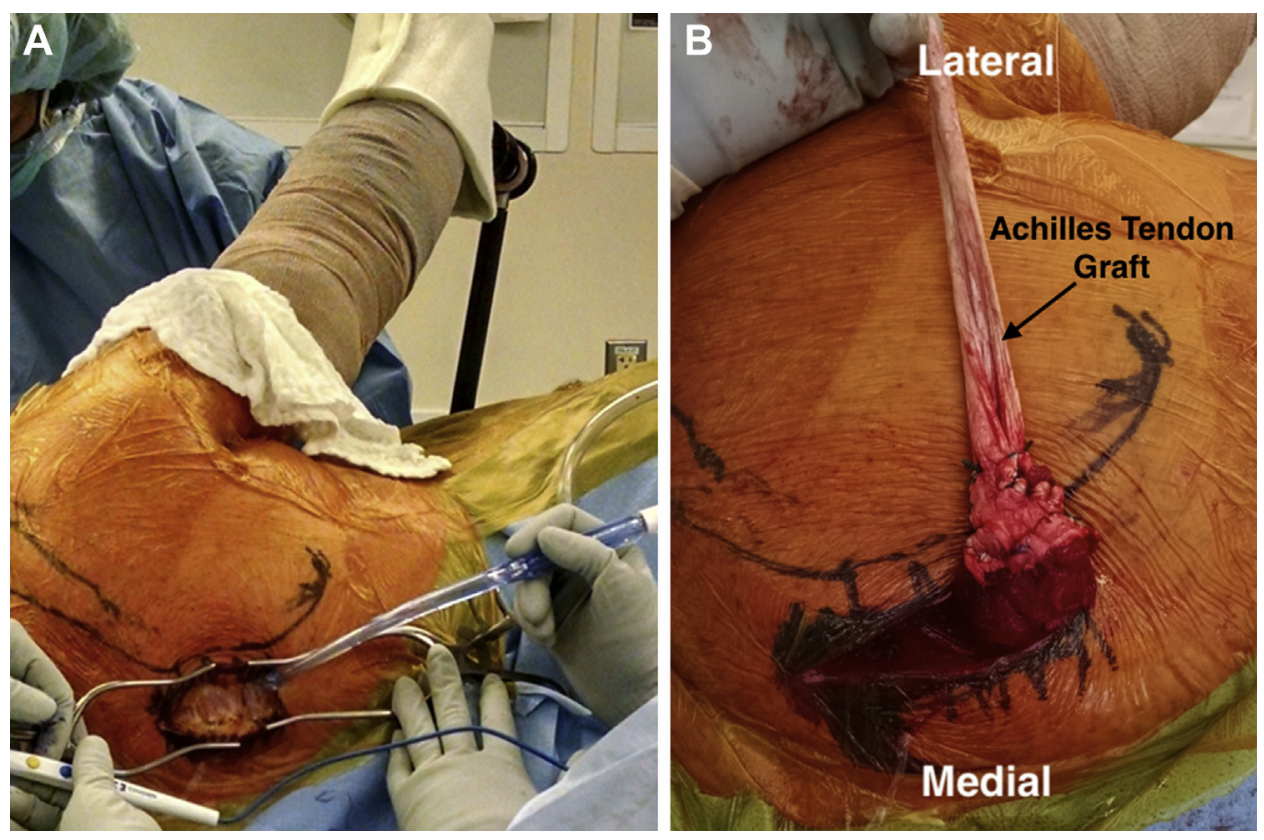

deltoid, the infraspinatus fascia was incised and opened at the horizontal incision. Through this medial opening, a switching stick was passed into the joint while viewing from an anterolateral portal. A large (8.25 or $10 \mathrm{~mm}$ ) cannula was placed over the switching stick and one limb of suture from each anchor was retrieved through the cannula and the cannula was removed (Fig 2B). The Achilles tendon allograft was prepared by placing a series of locking stiches into the tendon, terminating approximately $1 \mathrm{~cm}$ from the distal end. The retrieved sutures were then additionally passed through the Achilles tendon allograft in a locking-stitch fashion from distal to proximal. The 2 suture limbs were then tied to one another at the medial aspect of the graft. The graft was shuttled into the subacromial space to its final position on the greater tuberosity and the sutures were tied arthroscopically (Fig 3). Two additional suture anchors were placed at the lateral footprint to complete a double-row repair. The arm was positioned in $45^{\circ}$ abduction and $45^{\circ} \mathrm{ER}$, and the graft was tensioned and sutured to the native trapezius tendon in a Pulver-Taft weave with multiple nonabsorbable sutures. A standard skin closure was then performed.
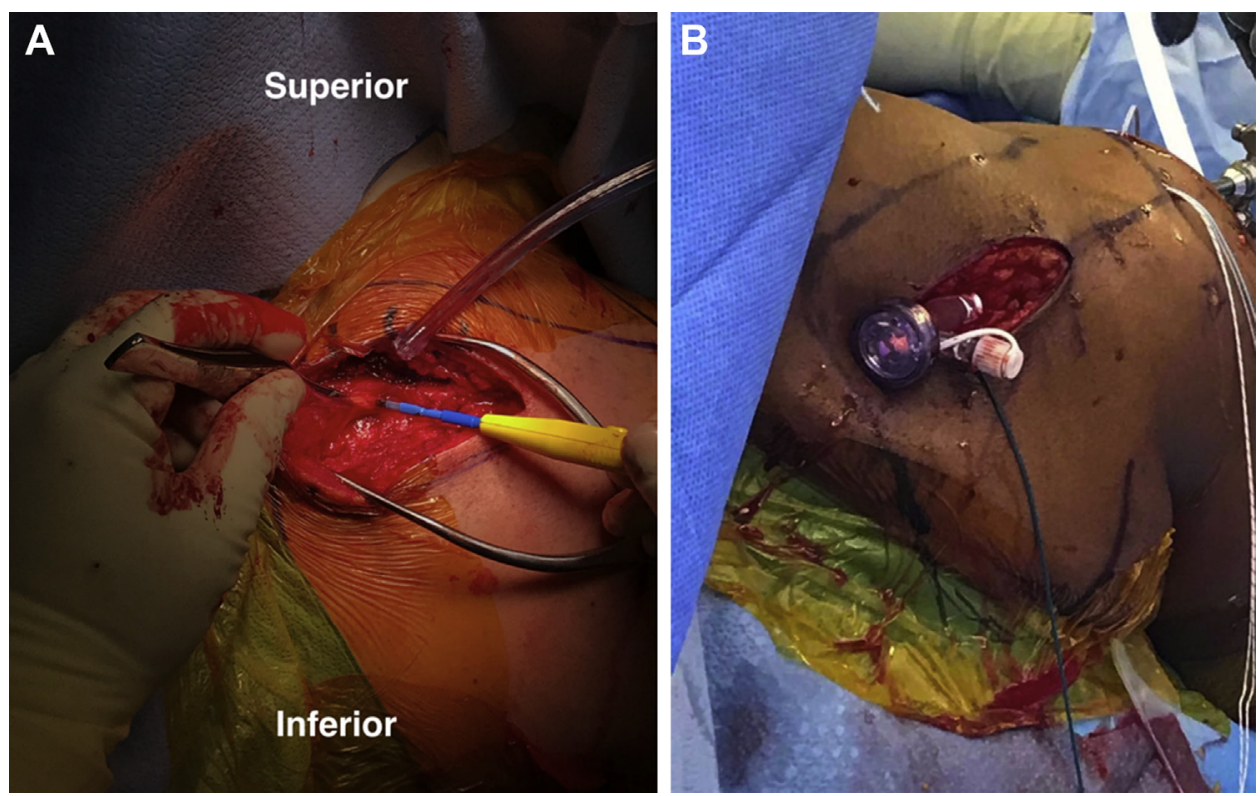

Fig 2. (A) Horizontal incision for a direct approach to the lower trapezius tendon (right shoulder). (B) Cannula placement for passage of the graft in the beach chair position. 


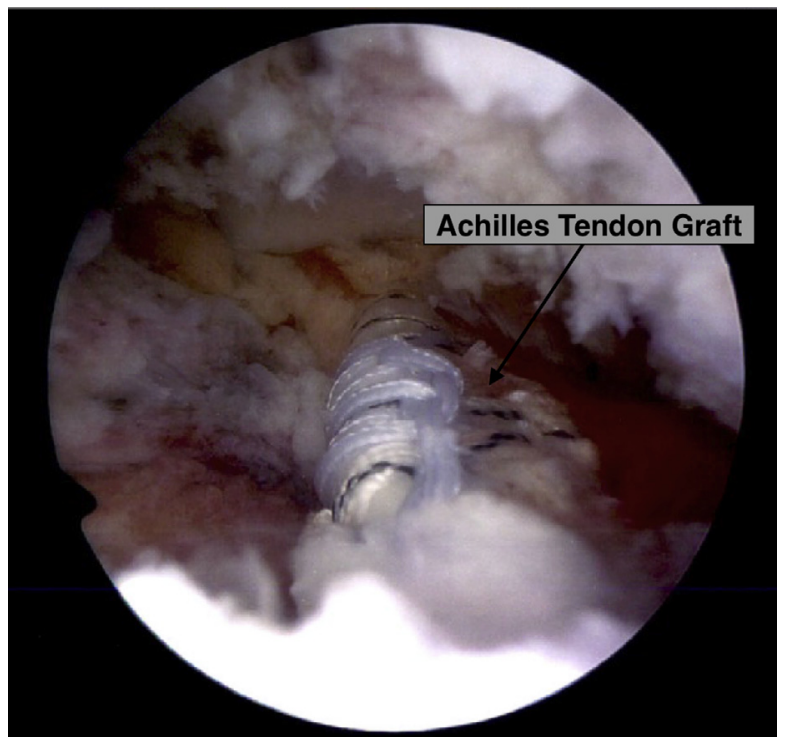

Fig 3. Arthroscopic view from the anterolateral portal showing repaired Achilles tendon allograft to the greater tuberosity with a double-row suture anchor construct (right shoulder).

\section{Postoperative Protocol}

The operative shoulder was placed in a gunslinger abduction brace at $30^{\circ}$ abduction and 30-60 ER for 6 weeks. The arm was taken out of the brace for supine FE exercises starting at 2 weeks after surgery. Formal physical therapy was initiated at 6 weeks after surgery. At weeks 6-12, ROM is initiated, restricting any crossbody adduction. At 12 weeks, isometric rotator cuff strengthening began as well as scapular conditioning. Isotonic strengthening began at 4 months and return to work and recreational activities were allowed at 6 months.

\section{Statistics}

Summary statistics, including means and standard deviations, were calculated. The Shapiro-Wilk test was used to determine normality of data. The Student $t$ test was used for comparison of means. Fisher exact test was used for categorical data, and linear regression was used to assess risk factors for revision. All statistics were performed using Stata software (StataCorp, College Station, TX). Significance was set as $P<.05$.

\section{Results}

Fifteen patients were included in the analysis, 14 of whom had previous rotator cuff surgery. Mean age was $52 \pm 7.3$ (range 31-62) years, 14 (93.3\%) were men, 13 $(92.9 \%)$ were manual laborers, and $9(60 \%)$ had a worker's compensation claim. Three patients $(20 \%)$ underwent conversion to RSA during the study period at a mean $23.7 \pm 11.5$ (range 12-35) months after the initial surgery and were not included in the functional outcome analysis. Of the remaining 12 patients, there were significant improvements in American Shoulder and Elbow Surgeons (43.2-77.2, $P=.0003)$, Single Assessment Numeric Evaluation (34.1-71.1, $P=.004$ ), Simple Shoulder Test (28.7-75.9, $P=.0005)$, and Veterans Rand Health Survey-physical score (36.1-46.6, $P=.005)$ at a mean follow-up of $24.1 \pm 9.6$ months (range 12-38.5 months) (Fig 4). Patients also demonstrated improvements in active FE $\left(98^{\circ}\right.$ to $144^{\circ}$, $P<.0001)$, abduction $\left(74^{\circ}\right.$ to $\left.127^{\circ}, P<.0001\right)$, and ER $\left(23^{\circ}\right.$ to $43^{\circ}, P<.0001$ ) (Fig 5 ).

On bivariate analysis, age, body mass index, smoking status, and open versus arthroscopic surgery were not correlated with failure or functional outcomes. However, reduced preoperative active $\mathrm{FE}$ and abduction were predictive of failure $\left(40^{\circ}\right.$ vs $113^{\circ}, P=.003 ; 27^{\circ}$ vs $\left.84^{\circ}, P=.01\right)$, respectively. Of the 4 patients with preoperative pseudoparesis (defined as active FE $<90^{\circ}$ ), all had their pseudoparesis reversed, but 3 were eventually converted to RSA due to poor strength and/ or continued pain due to progressive arthritis.

At final follow-up, all patients who had not "failed" their index surgery (with failure defined as reoperation or conversion to RSA) reported either being "very satisfied" $(66.7 \%)$ or "somewhat satisfied" (33.3\%) with their outcomes. Seven patients $(58.3 \%)$ had returned to full work duty, $3(25 \%)$ returned to modified duty, and $2(16.7 \%)$ were unable to return to work. Both patients unable to return to work and 2 of the 3 patients returning to modified duty were involved in worker's compensation claims. Figure 6A shows ultrasound image of an intact repair in one asymptomatic patient who was brought back to evaluate the integrity of the repair. There were no cases of nerve injury. Two patients who underwent the open technique had a superficial wound debridement for seroma and dehiscence of the posterior wound. One patient who was converted to RSA had multiple positive cultures at the time of revision for Cutibacterium acnes.

\section{Discussion}

Open or arthroscopic-assisted LTT for IRCT resulted in $80 \%$ survival at early follow-up, and improvements in both ROM and functional outcomes were seen. In addition, there was a reasonable rate of return to work in a population consisting mainly of laborers with a high number of worker's compensation claims. Preoperative FE appears to be an important predictor of outcomes and need for reoperation.

Three patients in this series were considered failures and underwent RSA, and 1 patient is likely to have RSA in the future. Preoperative pseudoparesis $\left(<90^{\circ}\right.$ of active FE) was present in all patients who failed LTT and underwent RSA. Elhassan et al. ${ }^{11}$ in a series of 33 patients undergoing LTT had no conversions to RSA reported at an average of 47-month follow-up. One 
Fig 4. Functional outcomes data. *indicates statistically significant difference $(P<.05)$. (ASES, American Shoulder and Elbow Surgeons; SANE, Single Assessment Numeric Evaluation; VAS, visual analog scale; VR12-P, Veterans Rand 12 Item Health Survey-Physical Evaluation, VR12-M, Veterans Rand 12 Item Health Survey-Mental Evaluation.)

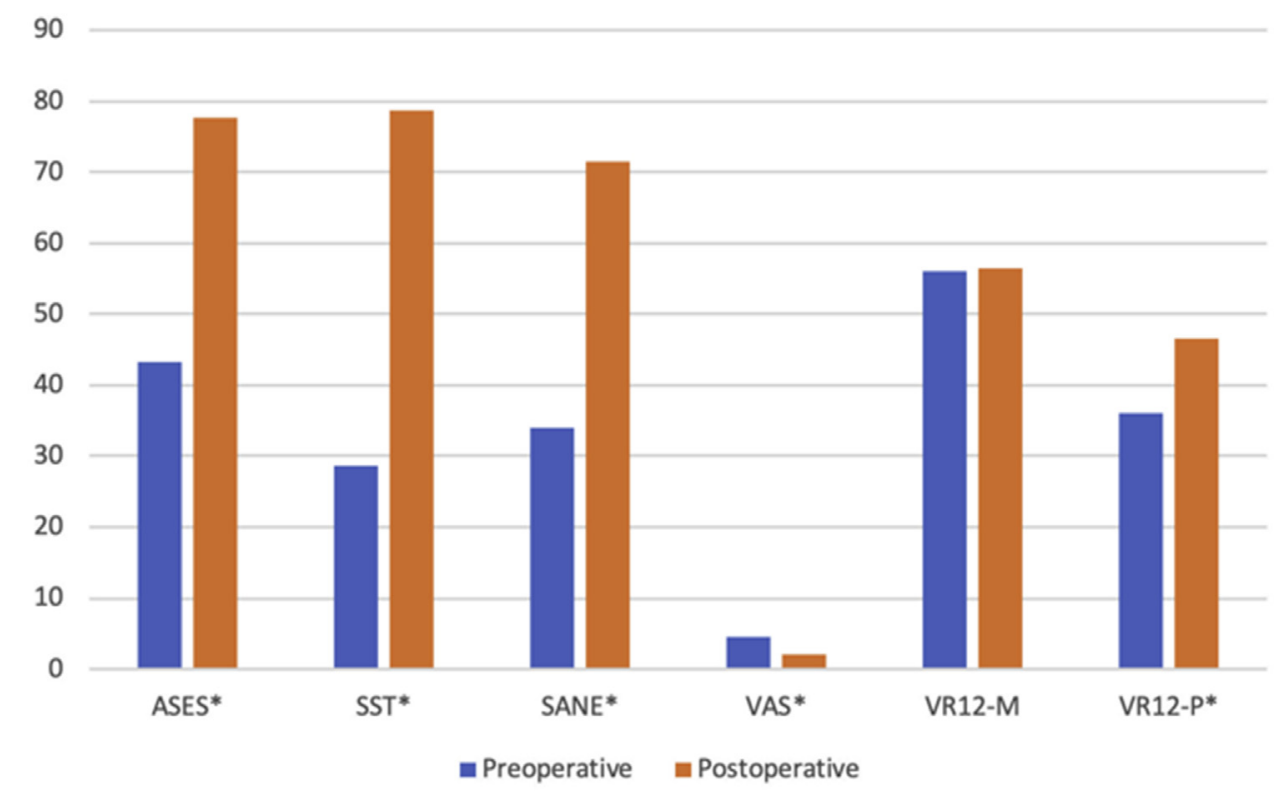

patient did have a glenohumeral fusion, however, due to complications related to a deep infection. The authors found that patients with $<60^{\circ}$ of preoperative active elevation had significantly lower gains in motion postoperatively than patients with $>60^{\circ}$ of preoperative active elevation. Our study results corroborate this notion that patients with lower preoperative active elevation are less likely to achieve desirable outcomes. In our current practice, we do not indicate patients with less than $90^{\circ}$ of active FE for isolated LTT.
In our cohort, there were significant improvements in functional outcomes and ROM from preoperative to postoperative measurements. Our ROM results show improvements in FE from $98^{\circ}$ to $144^{\circ}$, abduction from $74^{\circ}$ to $127^{\circ}$, and ER from $23^{\circ}$ to $43^{\circ}$ after LTT. Elhassan et al. $^{11}$ reported similar improvements in ROM including active $\mathrm{FE}\left(70^{\circ}\right.$ to $\left.120^{\circ}\right)$, abduction $\left(40^{\circ}\right.$ to $\left.90^{\circ}\right)$, and ER $\left(20^{\circ}\right.$ to $\left.50^{\circ}\right) .{ }^{11}$ Valenti et al. ${ }^{15}$ reported on 14 patients undergoing arthroscopic-assisted LTT with 2-year follow-up. ER improved from $-20^{\circ}$

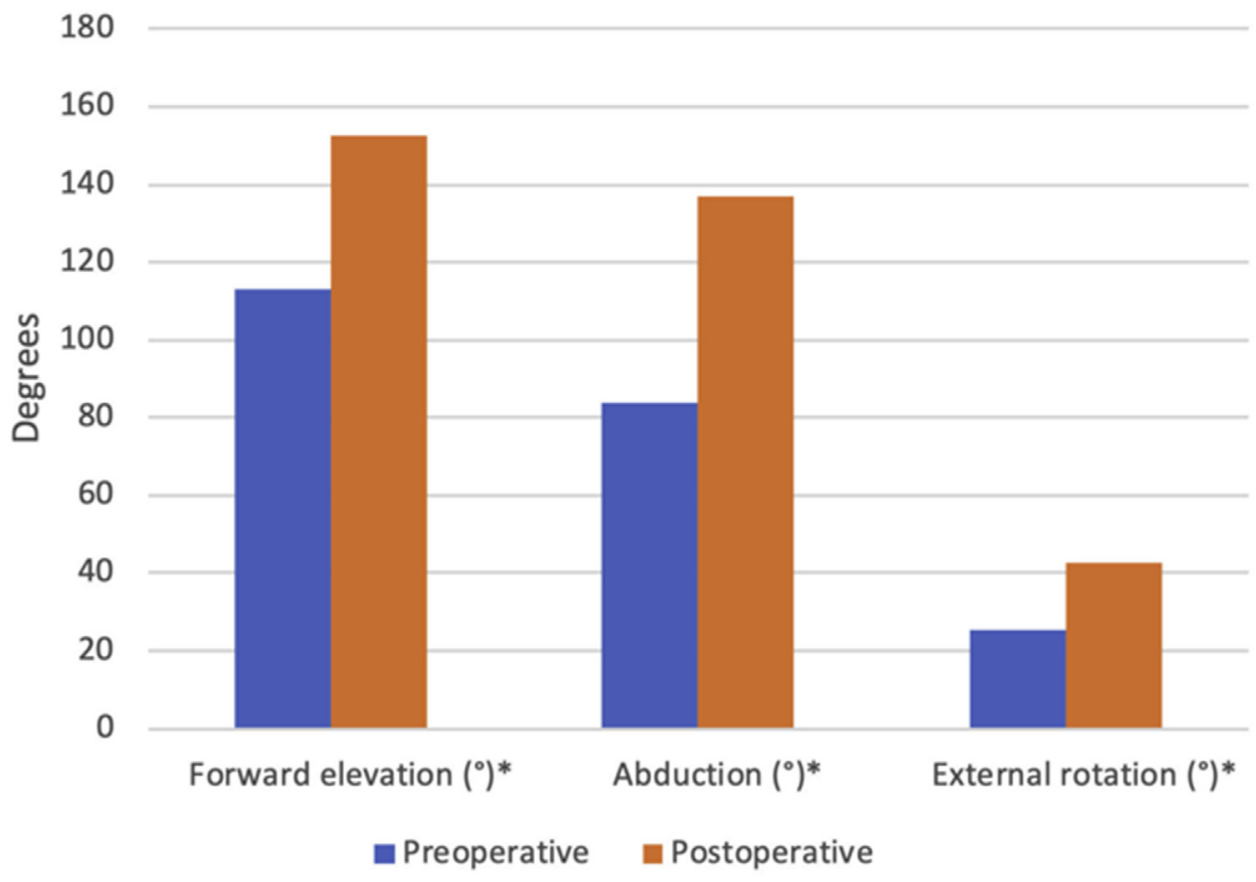

Fig 5. Range of motion outcomes. *Indicates statistically significant difference $(P<.05)$. 

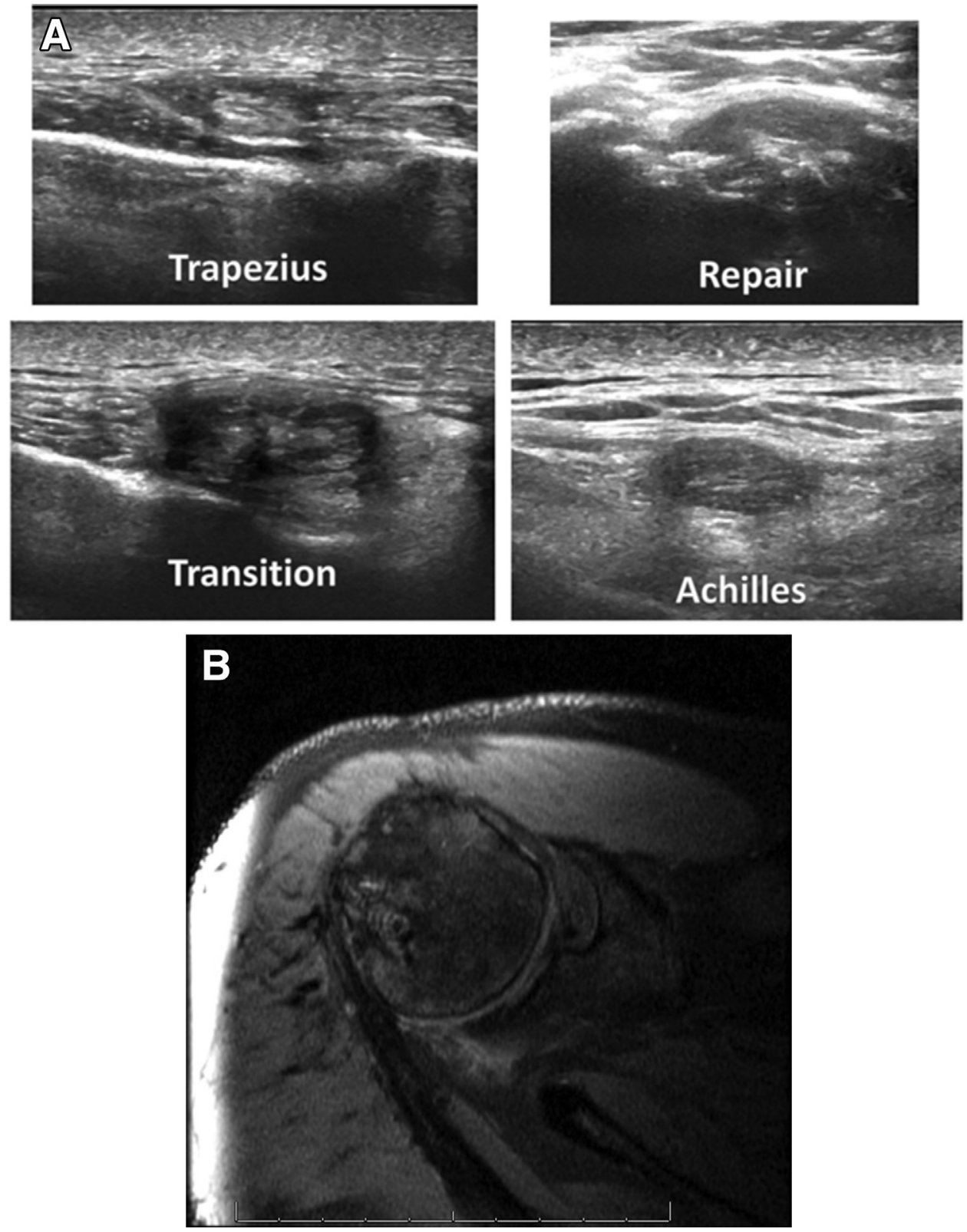

Fig 6. (A) Ultrasound of intact tendon transfer (right shoulder). (B) Magnetic resonance imaging of patient with loss of active elevation with a structurally intact repair. preoperatively to $24^{\circ}$ postoperatively, and FE improved from $150^{\circ}$ preoperatively to $160^{\circ}$ postoperatively.

Our results also show that the majority of patients were able to return to work, mostly at full duty, although nearly $17 \%$ were unable to return. It is important to note, however, that patients with work restrictions were more commonly involved in worker's compensation claims $(80 \%)$ compared with those who returned to full duty $(42.9 \%)$. Although tendon transfers are historically performed in greater-demand manual laborers, there is surprisingly limited outcomes data evaluating return-to-work rates. In our estimation, this is a critical outcome variable that should be consistently reported in future studies. These types of data provide both patient and physicians with a means of shaping preoperative expectations of this procedure.

Two patients who had an open technique underwent a superficial wound debridement for seroma and wound breakdown. Seroma formation also occurred in 4 patients $(12 \%)$ in the study by Elhassan et al. ${ }^{11}$ Although these 4 patients were managed nonoperatively with no sequelae, our subjects opted for surgical treatment based on surgeon and patient preference, resulting in successful treatment. With the development of the arthroscopic-assisted technique, it is possible that this will result in fewer wound complications. No patients in our study who underwent an arthroscopic-assisted technique had a wound complication. Valenti et al. ${ }^{15}$ reported 2 complications out of 
their cohort of 14 arthroscopic-assisted LTT procedures. Both involved hematoma at the harvest site of the lower trapezius, and 1 of the 2 patients required open debridement due to Cutibacterium acnes infection.

\section{Limitations}

The results of this study should be considered in light of the following limitations. The retrospective nature creates a selection bias in which patients were indicated for LTT by the treating surgeon. As discussed, there are a number of treatment possibilities for these tears, none of which has shown clear superiority. Magnetic resonance imaging scans also was not available for review, and therefore we were unable to control for Goutallier stage. ${ }^{16}$ Two different techniques were used (open and arthroscopic-assisted); however, we believe that this demonstrates both the evolution of this technique as well as the early learning curve that many surgeons will experience. In addition, unblinded measurements were not made with a goniometer, strength measurements were not taken, and follow-up was short at 1 year from surgery. Lastly, outcomes were not compared to a control group; therefore, patients were instead compared with their preoperative functional status.

\section{Conclusions}

LTT results in successful clinical outcomes with a high rate of return to work in a challenging patient population. However, only $67 \%$ of patients rated themselves as "very satisfied," and $20 \%$ of patients were revised to RSA before 1 year. Limited preoperative active forward elevation $\left(<90^{\circ}\right)$ appears to predict poor functional results and risk for reoperation.

\section{References}

1. Bedi A, Dines J, Warren RF, Dines DM. Massive tears of the rotator cuff. J Bone Joint Surg Am 2010;92:1894-1908.

2. Mulieri P, Dunning P, Klein S, Pupello D, Frankle M. Reverse shoulder arthroplasty for the treatment of irreparable rotator cuff tear without glenohumeral arthritis. J Bone Joint Surg Am 2010;92:2544-2556.

3. Fenlin JM Jr, Chase JM, Rushton SA, Frieman BG. Tuberoplasty: Creation of an acromiohumeral articulation-a treatment option for massive, irreparable rotator cuff tears. J Shoulder Elbow Surg 2002;1 1:136-142. doi: $10.1067 / \mathrm{mse} .2002 .121764$.

4. Heuberer PR, Kolblinger R, Buchleitner S, et al. Arthroscopic management of massive rotator cuff tears:
An evaluation of debridement, complete, and partial repair with and without force couple restoration. Knee Surg Sports Traumatol Arthrosc 2016;24:3828-3837.

5. Gartsman GM. Massive, irreparable tears of the rotator cuff. Results of operative debridement and subacromial decompression. J Bone Joint Surg Am 1997;79: 715-721.

6. Mihata T, McGarry MH, Pirolo JM, Kinoshita M, Lee TQ. Superior capsule reconstruction to restore superior stability in irreparable rotator cuff tears: A biomechanical cadaveric study. Am J Sports Med 2012;40:2248-2255.

7. Gerber C. Latissimus dorsi transfer for the treatment of irreparable tears of the rotator cuff. Clin Orthop Relat Res 1992;275:152-160.

8. Iannotti JP, Hennigan S, Herzog R, Kella S, Kelley M, Leggin B, et al. Latissimus dorsi tendon transfer for irreparable posterosuperior rotator cuff tears. Factors affecting outcome. J Bone Joint Surg Am 2006;88: 342-348.

9. Omid R, Heckmann N, Wang L, McGarry MH, Vangsness CT Jr, Lee TQ. Biomechanical comparison between the trapezius transfer and latissimus transfer for irreparable posterosuperior rotator cuff tears. J Shoulder Elbow Surg 2015;24:1635-1643.

10. Hartzler RU, Barlow JD, An KN, Elhassan BT. Biomechanical effectiveness of different types of tendon transfers to the shoulder for external rotation. J Shoulder Elbow Surg 2012;21:1370-1376.

11. Elhassan BT, Wagner ER, Werthel JD. Outcome of lower trapezius transfer to reconstruct massive irreparable posterior-superior rotator cuff tear. J Shoulder Elbow Surg 2016;25:1346-1353.

12. Clouette J, Leroux T, Shanmugaraj A, Khan M, Gohal C, Veillette C, et al. The lower trapezius transfer: A systematic review of biomechanical data, techniques, and clinical outcomes. J Shoulder Elbow Surg 2020;29:1505-1512.

13. Elhassan BT, Alentorn-Geli E, Assenmacher AT, Wagner ER. Arthroscopic-assisted lower trapezius tendon transfer for massive irreparable posterior-superior rotator cuff tears: Surgical technique. Arthrosc Tech 2016;5: e981-e988.

14. Wagner ER, Woodmass JM, Welp KM, et al. Novel arthroscopic tendon transfers for posterosuperior rotator cuff tears: Latissimus dorsi and lower trapezius transfers. JBJS Essent Surg Tech 2018;8:e12.

15. Valenti P, Werthel JD. Lower trapezius transfer with semitendinosus tendon augmentation: Indication, technique, results. Obere Extrem 2018;13:261-268.

16. Goutallier D, Postel JM, Bernageau J, Lavau L, Voisin MC. Fatty muscle degeneration in cuff ruptures. Pre- and postoperative evaluation by CT scan. Clin Orthop Relat Res 1994;304:78-83. 